\title{
Sports Scientific Consideration of Soccer Penalty Kicks
}

\author{
Chong-hoon Lee ${ }^{1}$ \\ ${ }^{1}$ Department of Sports Science, Seoul National Univ. of Science \& Tech., \\ Seoul, Korea \\ ${ }^{1}$ leejh36@snut.ac.kr
}

\begin{abstract}
Soccer is a popular sport that has the largest number of fans throughout the world to the extent that the number of member countries of the Fédération Internationale de Football Association has reached 204 (as of 2014) and is one of national sports frequently played by numerous players and club members. The present study was conducted to help soccer related persons make more accurate and efficient penalty kicks by investigating and generalizing the data on variables that affect major kinesiological factors of penalty kicks. The conclusions obtained through previous studies are as follows. Information on run-ups provides clues to determine the kicking methods of the players that make penalty kicks and ball directions and can enable the goalkeeper to predict the timing and direction of balls. The kicker's supporting foot was closed so that the outside of the foot is seen when seen from the viewpoint of the goalkeeper in most cases. When the pelvis is tilted rightward, the probability for the ball to go leftward is high and the opposite is the same as well. Shoulder angles are helpful for the prediction of ball directions in advance because ball directions can be predicted at the moment when the kicking foot reached the highest height already before the moment of kicking through shoulder tilt angles. It can be seen that kickers generally make penalty kicks with the trunk a little tilted.
\end{abstract}

Keywords: Penalty kick, Approach angle, Supporting foot angle, Pelvis angle, Shoulder angle, Trunk angle

\section{Introduction}

Soccer is a popular sport that has the largest number of fans throughout the world to the extent that the number of member countries of the Fédération Internationale de Football Association has reached 204 (as of 2014) and is one of national sports frequently played by numerous players and club members.

Soccer is a sport that requires agility and the ability to react instantly, which are anaerobic capacities of the body, and cardiopulmonary endurance, which is an aerobic capacity (Yu Yeon-Sik, 1996), and involves personal skills, organizing ability, tactical understanding, and mental power elements such as fighting spirit. Penalty kicks are psychological confrontation between the kicker and the goalkeeper to determine the outcome of the game in a special situation where the ball is placed at the point $11 \mathrm{~m}$ away from the goal, in which the physical skill of the kicks and strong pressure should be overcome. In general, there are two types of penalty kicks used in soccer. One type is given against fouls occurring during the game and the other type is used to determine the outcome of the game when a game ended in a tie, which is called penalty shoot-out. The penalty kicks given against fouls and the penalty shoot-out used to determine the outcome are the same in their situations that are one on one confrontation between the keeper and kicker as a tense war of nerves. However, penalty shoot-out is different from foul penalty kicks in that the strategy of the kicking team and the mental state of the kicker act as decisive keys to determine the outcome. In the 2012 London Olympics, South Korea 
achieved a record of advancing to the round of 14 by defeating the UK, the suzerain of soccer with a penalty kick outcome. As such, in soccer games, penalty kicks have become a very important skill to determine the outcome (So Jae-Mu et al., 2013). According to an analysis of the moment at which a penalty kick is made conducted in terms of time, the time taken by the ball to go into the goal after being kicked is $0.5 \mathrm{sec}$. on average and the time taken by a world-class goalkeeper to react after kicking is $0.75 \mathrm{sec}$. Therefore, if the kicker kicks the ball accurately, the goalkeeper cannot block the ball no matter how accurately he predicted the direction of the ball (Lee Jong-Hun 2014). If the kick is made accurately, the goalkeeper can never win. A researcher presented the following four conditions as penalty kick success conditions; a run-up of 5 6 steps from the end of the penalty box, the angle of the kicker's approach to the ball in a range of $20 \sim 30^{\circ}$, a ball speed not lower than $100 \mathrm{~km} / \mathrm{h}$, and the direction of the ball to go to a point $50 \mathrm{~cm}$ away from the goal post. If a penalty kick is made to satisfy these conditions, the penalty kick cannot be blocked by anybody whatsoever. Although kinematic, kinetic and psychological analyses of soccer kicks have been continuously conducted (Yun Jae-Man, 2000; Kim Eui-Hwan et al., Kim Seong-Ho 2003; Jin Yeong-Hwan et al. 2005), despite that penalty kicks are an important factor that can have large effects on outcomes, and studies on penalty kicks are insufficient. This is considered attributable to the fact that the kicking methods used for penalty kicks are not peculiars ones not used in normal games but are the same ones as those frequently used during normal games (Shin Je-Min 2005). However, unlike general kicks, penalty kick is made in a unique situation where the game time is stopped and the kicker confronts one on one with the goalkeeper at a defined distance of $11 \mathrm{~m}$. In addition, since only one penalty kick may determine the outcome of the game, more serious and in-depth studies on penalty kicks are considered necessary. If the physical mechanism and psychological factors of penalty kicks are understood from the viewpoint of sports, proper skills and strategies to be used can be provided to players that act in the field, trainers, coaches, and game analyzers. The present study was conducted to help soccer related persons make more accurate and efficient penalty kicks by investigating and generalizing the data on variables that affect major kinesiological factors of penalty kicks

\section{Result}

\subsection{Kinetic Mechanism of Kicks}

For kickers to enhance shooting success rates, the understanding of the principle of kicking motions and kinesiological structures is important. To kinetically analyze the basic motions of kicks, first, take a step forward with the supporting leg and rotate the pelvis of the kicking leg backward to apply a strain to the thigh. Thereafter, bend the hip and the knee simultaneously to the kicking leg forward while applying a momentary strain to the lower leg of the kicking leg. Then, momentarily stop the lower leg moving forward and upward and apply a strain to the lower leg until the foot hits the ball to make a powerful kick while the opposite side arm swings forward as a 'reaction to the motion.' The foregoing are motions for individual segments of the human body to move sequentially to increase the speed of the foot, which is the most distal segment, and are a method of using the principle of whip-like motions. Although irregular motions are often attempted to deceive the goalkeeper but such motions are not normal postures and are not appropriate to be generalized.

\subsection{Experimental Tool}

The following experimental equipment (Kinetic, Kinematic variable) was used for motion analysis. 


\subsubsection{Kinetic Equipment}
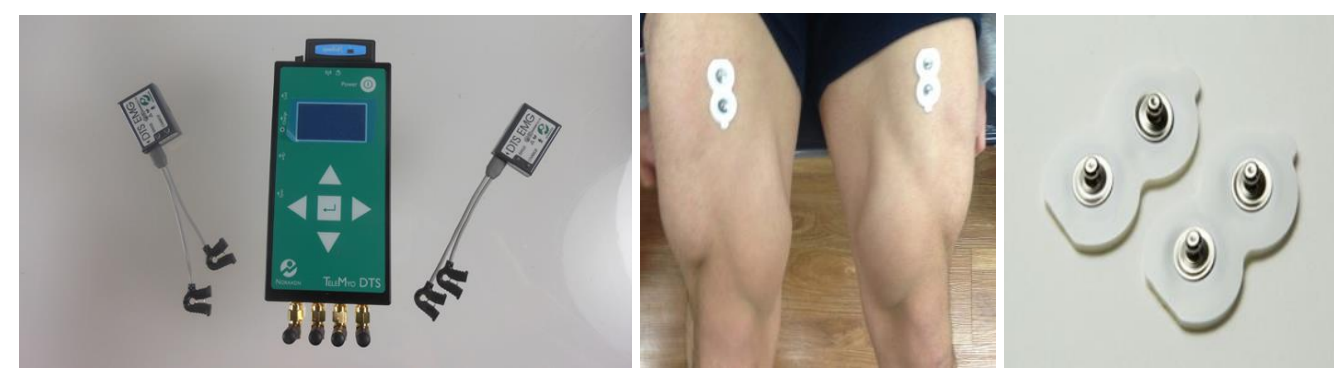

Figure 1. Electromygraphy
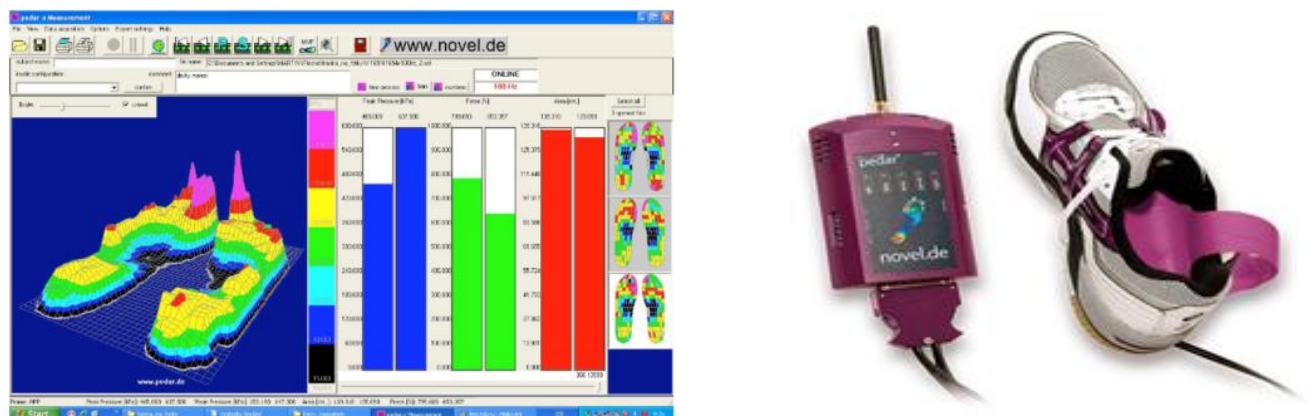

Figure 2. Pedar
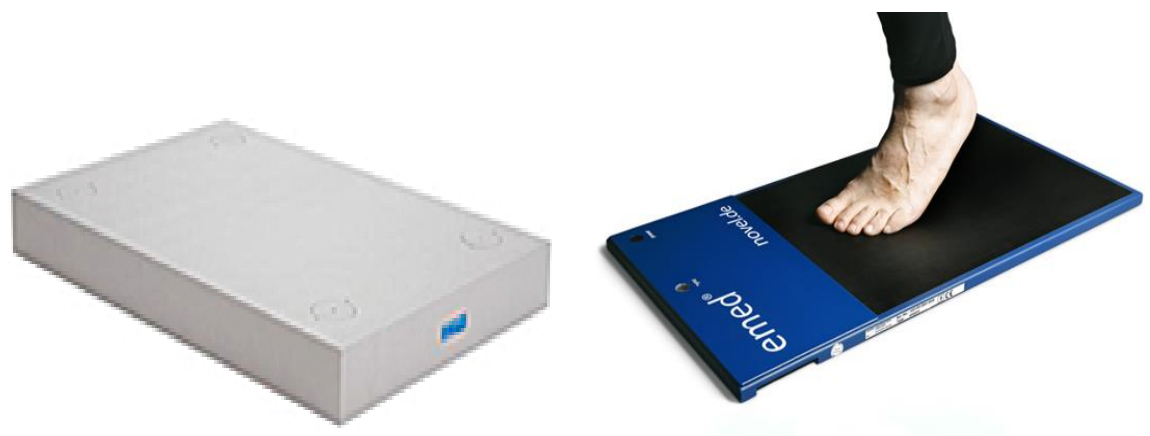

Figure 3. Force Plate

2.2.2. Kinematic Equipment
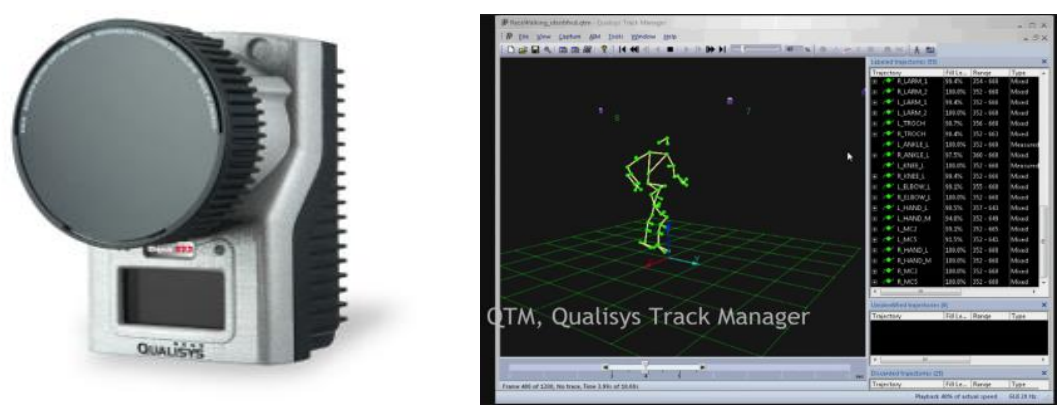

Figure 4. Qualisys 

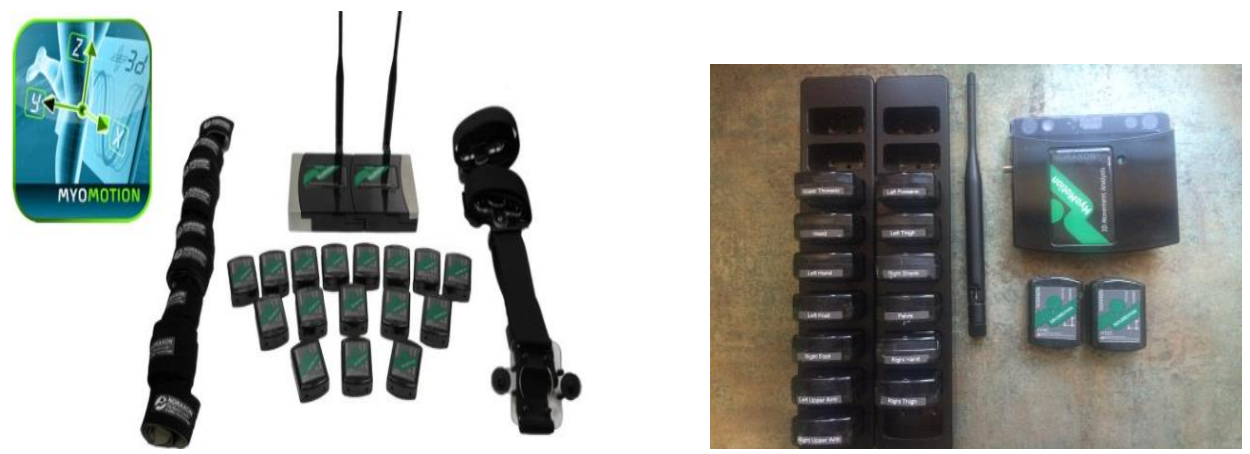

Figure 5. Inertial Measurement Unit

\subsection{Approach Angle}

Run-up is a preliminary motion used not only in soccer kick motions but also in field events of athletics such as the long jump, the triple jump, and the pole vault to produce the maximum effect by adjusting the step, time, stride length, and stride frequency before performing the main motion and the angle of the approach is very important for the athletic performance. As shown in Figure 6, the approach angle (A) is the angle made by the vector formed by the center point of the two feet of the kicker when the kicker starts to make a shooting and the ball and the Y-axis. Lee Gi-Cheong et al. (2008) analyzed approach angles with high school players and university players to examine the relationship between the direction of the kicker's run to kick the ball and the direction of the ball movement after kicking. The approach angles of the high school players and university players were shown to be approximately $27 \pm 8.1^{\circ}$ when the ball was kicked toward the left top of the goal, approximately $28 \pm 4.4^{\circ}$ in the case of the center top, approximately $32 \pm 12.4^{\circ}$ in the case of the right top, approximately $26 \pm 11.5^{\circ}$ in the case of the left bottom, approximately $22 \pm 8.4^{\circ}$ in the case of the center bottom, and approximately $36 \pm 10.3^{\circ}$ in the case of the right bottom. That is, it can be seen that, when seen from the viewpoint of the goalkeeper, right footed players started from the right side of the goalkeeper to make the penalty kick. When seen from the viewpoint of the goalkeeper, it can be seen that approach angles were different between when the ball was kicked toward the left top of the goal and when the ball was kicked toward the left bottom of the goal and between when the ball was kicked toward the center bottom of the goal and when the ball was kicked toward the bottom of the left side of the goalkeeper. In addition, Lim Bi-Oh et al. (2002) reported that run-up angles were shown to be larger when the ball was kicked rightward than when kicked leftward and when the ball was kicked to fly high than when the ball was kicked to fly low close to the ground regardless of whether kicked rightward or leftward but the differences were not statistically significant. Since the kicker determines the direction of the ball and the method of kicking in advance before kicking when the goalkeeper does not move in advance, approach angles are determined according to complex factors such as the kicking method, ball speed, the number of spins of the ball suitable for the characteristics of individual players and game environments and maintaining certain patterns based on the foregoing is considered effective. 


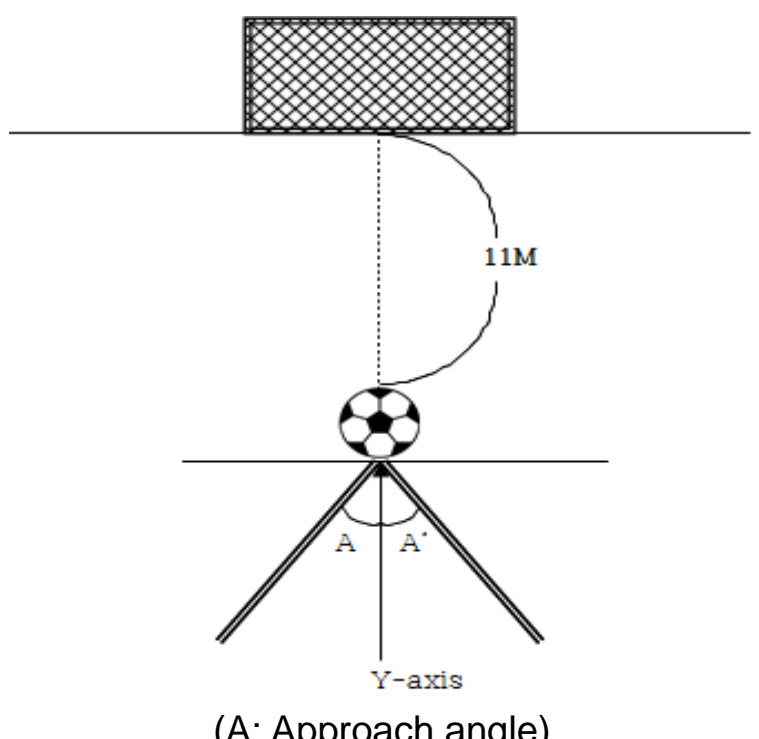

Figure 6. Approach Angle

\subsection{Changes in Steps}

To examine the relationship between changes in steps when the kicker runs to kick the ball and the flying direction of the ball, the lengths of kicker's steps immediately before kicking and at the moment of kicking were analyzed.

As for changes in the kicker's steps, the lengths of the steps immediately before making a penalty kick were shown to be approximately $90 \pm 20.2 \mathrm{~cm}$ when the ball was kicked toward the left top of the goal, approximately $97 \pm 19.4 \mathrm{~cm}$ in the case of the center top, approximately $95 \pm 16.8 \mathrm{~cm}$ in the case of the right top, approximately $102 \pm 18.7 \mathrm{~cm}$ in the case of the left bottom, approximately $110 \pm 21.8 \mathrm{~cm}$ in the case of the center bottom, and approximately $100 \pm 23.0 \mathrm{~cm}$ in the case of the right bottom. The lengths of the steps at the moment of kicking were shown to be approximately $158 \pm 25.1 \mathrm{~cm}$ when the ball was kicked toward the left top of the goal, approximately $162 \pm 24.8 \mathrm{~cm}$ in the case of the center top, approximately $161 \pm 25.5 \mathrm{~cm}$ in the case of the right top, approximately $164 \pm 15.9 \mathrm{~cm}$ in the case of the left bottom, approximately $159 \pm 21.3 \mathrm{~cm}$ in the case of the center bottom, and approximately $160 \pm 22.0 \mathrm{~cm}$ in the case of the right bottom. (Lee Gi-Cheong et al., 2008).

Given the foregoing results, it can be seen that the players increased their step length by approximately $60 \mathrm{~cm}$ at the moment of making a penalty kick compared to immediately before making a penalty kick. This is considered attributable to the fact that to make more powerful shooting, the players maximally increased the extension and flexion of the joints of segments of their bodies thereby making large swing motions. This pattern was shown to have been maintained almost constantly regardless of ball directions.

In a study conducted with university students, Lim Bi-Oh et al. (2002) reported that the steps lengths when the supporting foot was landing finally for kicking were $84.49 \pm 6.41 \%$ of the height of the kicker when the ball flied low rightward, $85.35 \pm 8.99 \%$ when the ball flied high rightward, $87.32 \pm 6.17 \%$ when the ball flied low leftward, and $84.40 \pm 5.19 \%$ when the ball flied high leftward.

\subsection{Time Required}

From the viewpoint of goalkeeper that has to block the ball from penalty kicks, constant run-up speeds are more advantageous for him to catch the timing. Therefore, the kicking player may change the run-up speed to cause difficulties for the goalkeeper in catching the timing in order to increase the probability to score a goal. Although a run-up 
for a long distance is advantageous in that it can increase acceleration for kicking motion so that the ball speed can be increased, it causes difficulties in maintaining the rhythm and the step at the time of kicking motion. Recently, there has been a tendency to run up in a short distance probably because it is more advantageous from the viewpoint of kicking player in preventing the goalkeeper from catching the timing by reducing the time for the goalkeeper to collect information and predict the trajectory of the ball. Run-up speeds were shown to be higher when the kicker was kicking leftward then when kicking rightward, when kicking the ball to fly low than when kicking the ball to fly high regardless of whether the ball was kicked to fly rightward or leftward (Lim Bi-Oh et al., 2002). In a study conducted with university players, So Jae-Mu et al. (2013) reported that the time taken for motions from the landing of the supporting foot to the follow through after kicking was $0.29 \pm 0.01 \mathrm{~s}$. In a study where ball speeds after were examined, kicking Lee Gi-Cheong et al. (2008) reported that players did not change their kicking speeds according to ball directions but maintained the kicking speeds constantly when they were making penalty kicks and that the speeds were more accurately maintained constantly in the case of higher level players.

\subsection{Angle Factor}

The angles made by major segments of the trunk and lower limbs of the kicker when the kicking foot hits the ball after stepping on the ground with the supporting foot and taking a series of motions and the definitions of the angles are as follows. The directions were set after setting an inertial axis based on the reference scale and the anteroposterior direction from the viewpoint of the kicker looking at the goal was defined as Y-axis, the lateral direction as $\mathrm{X}$-axis, and the direction perpendicular to the horizontal plane as $\mathrm{Z}$ axis. As can be seen in <Figure 7>, the supporting foot's angle (B) is the angle formed by the supporting foot and the Y-axis immediately before shooting, the pelvic tilt angle $(\mathrm{C})$ indicates the angle formed by the horizontal plane and the pelvis, the shoulder tilt angle (D) indicates the angle formed by the horizontal plane and the shoulder line, and the trunk tilt angle (E) refers to the angle formed by the vertical axis and the trunk.
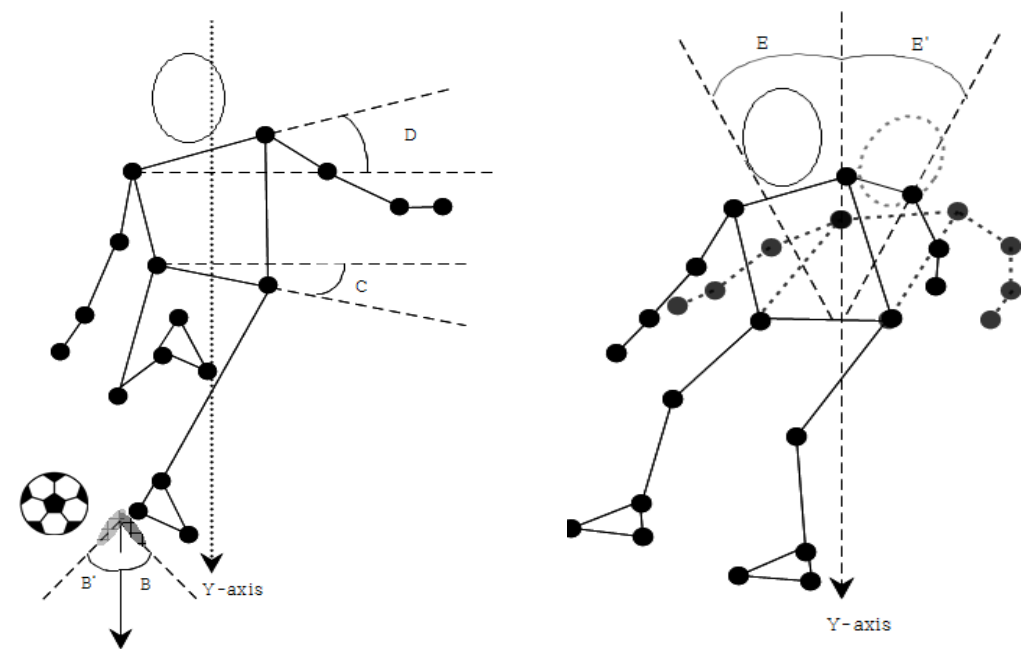

(B: Supporting foot angle, C: Pelvis angle, D: Shoulder angle, E: Trunk angle)

Figure 7. The Definition of an Angle

2.6.1. Supporting foot's Angle: The supporting foot's angle (B) was analyzed to examine the relationship between the supporting foot's angle at the moment when the kicker kicks the ball and the ball's flying direction. In a study conducted with high school players and university players, Lee Gi-Cheong et al.(2008) reported that the kicker's supporting foot angles were shown to be approximately $-19 \pm 15.1^{\circ}$ when the ball was 
kicked toward the left top of the goal, approximately $-11 \pm 8.2^{\circ}$ in the case of the center top, approximately $-20 \pm 7.4^{\circ}$ in the case of the right top, approximately $-5 \pm 16.4^{\circ}$ in the case of the left bottom, approximately $-5 \pm 27.5^{\circ}$ in the case of the center bottom, and approximately $-24 \pm 9.9^{\circ}$ in the case of the right bottom. That is, it can be said that, when seem from the viewpoint of the goalkeeper, penalty kicks were made with the supporting foot closed a little. It can be said that, when seem from the viewpoint of the goalkeeper, the kicker's supporting foot angles were different between when the ball was kicked toward the center top and when the ball was kicked toward the left bottom of the goal. Lim Bi-Oh et al. (2001) indicated that the direction angle of the supporting foot was located clockwise from the Y-axis, that is, rightward when the ball was kicked rightward and located counterclockwise from the Y-axis when the ball was kicked leftward. The goalkeeper can predict the direction of the ball to some extent by watching the direction of the kicker's supporting foot and catching the time can be sought as a way to reduce the kicking team's score.

2.6.2. Pelvic Angle: Lee Gi-Cheong et al. reported that pelvic tilt angles were investigated to examine the relationship the tilt angle $(\mathrm{C})$ formed by the pelvis and the horizontal plane at the moment when the kicker kicks the ball's flying direction and according the results, the pelvic tilt angles were shown to be approximately $19.85 \pm 5.98^{\circ}$ when the ball was kicked toward the right area of the goal, approximately $21.93 \pm 6.64^{\circ}$ when the ball was kicked toward the center of the goal, and approximately $35.20 \pm 6.84^{\circ}$ when the ball was kicked toward the left area of the goal(Lee Gi-Cheong et al., 2014). When seem from the viewpoint of the goalkeeper, it can be seen that the kickers kicked the ball in postures as tilted rightward. Pelvic tilt angles according to kicking methods were examined and according to the results, the pelvic tilt angles were shown to be the largest with a mean value of $27.8 \pm 5.9^{\circ}$ in the case of inside right kicks. This means that the pelvis turns clockwise around the major axis (Z-axis). Therefore, since the pelvis turns rightward when the ball is kicked rightward by an inside kick, the goalkeeper can predict the direction in advance to increase the probability to block the ball. Although the degrees of pelvic tilting can be predicted and standardized according to kicking methods and ball directions, since the pelvic tilt angles may vary with ball speeds and the number of spins of the ball at the moment of ball hitting, additional studies and discussions are required.

2.6.3. Shoulder Angle: The shoulder angle refers to the tilt angle (D) made by the shoulder line in relation to the horizontal plane at the moment when the kicker makes a shoot. In a study conducted with elementary school players, Lee Gi-Cheong et al. (2014) reported that when seen from the viewpoint of the goalkeeper, the shoulder angle were shown to be $34.61 \pm 8.22^{\circ}$ when the ball was kicked toward the right areas of the goal, $34.35 \pm 9.86^{\circ}$ in the case of the center, and $46.84 \pm 10.47$ in the case of the left area. When seen from the viewpoint of the kicker, it can be said that when the kicker wishes to send the ball rightward using his right foot, the shoulder tilt angle shows significant differences from other ball directions. Plagenhoef (1970) stated that the principle of kicking could be applied to use the kinetic energy of the trunk and that it is closely related with the interactions between the foot and the ground and between the supporting leg/the thigh and the trunk. Lee Gi-Cheong et al. indicated that shoulder angles are more helpful than pelvic angle for the prediction of ball directions in advance (Lee Gi-Cheong et al., 2014) because ball directions can be predicted at the moment when the kicking foot reached the highest height already before the moment of kicking through shoulder tilt angles. Whereas the pelvic tilt angle is determined at the final moment of shooting since the ball is kicked using the turning force obtained while the pelvis is turned, the shoulder angle can be said to be already determined during the run-up. The goalkeeper can watch the kicker's shoulder angle to predict the ball direction and comprehensively judge the posture of the kicker at the moment of kicking to block ball. 
2.6.4. Trunk's Anteversion/Retroversion Angles: The anteversion/retroversion angles of the trunk mean the angles (E) formed by the trunk of the kicker and the major axis (Zaxis). On reviewing the results of previous studies, it can be seen that kickers generally make penalty kicks with the trunk a little tilted and that anteversion/retroversion angles are shown to be larger during inside left kicks and in-step left kicks than during right inside/in-step kicks. The anteversion/retroversion angles of the trunk can be said to be closely related to the differences in the anthropotomical structures of kicking players, the positions and angle distances of the supporting foot and the ball, and ball directions. In addition, the angles may vary with the position of contact between the ball and the foot determined by kicking methods. The trunk tilt angle is determined by the angle of the kicking foot at the moment of hitting the ball, which can be said to affect the angle formed by the kicking foot and the ball.

\section{Conclusion}

In soccer games, penalty kicks are very important skills that determine the outcomes. The matters considered by the goalkeeper at the moments of penalty kicks can be said to be temporal factors and angle factors. Among the temporal factors, the moment of the landing of the supporting foot and the moment of ball hitting and among changes in the angles of human body segments, the angles of the supporting foot and the positions of the kicking leg, the trunk, and the shoulder are important variables. Although many researchers have conducted diverse studies for successful penalty kicks, more accurate and systematic data obtained through scientific verification are not sufficient. The conclusions obtained through previous studies are as follows.

(1) Information on run-ups provides clues to determine the kicking methods of the players that make penalty kicks and ball directions and can enable the goalkeeper to predict the timing and direction of balls. Kickers tend to start from the right side to make a leftward shoot, show a tendency to kick leftward when run-up angles are small while kicking rightward when run-up angles are large. In other words, when the kicker approaches almost in parallel to the ball, it can be said that the kicker is highly likely to kick straightforward or wind the ball with the inside of the foot before kicking. As for speed factors, since constant speeds are advantageous from the viewpoint of the goalkeeper as he can more easily catch the timing, the kicker can change run-up speeds to cause difficulties for the goalkeeper in catching the timing to increase the probability of successful shooting.

(2) The kicker's supporting foot was closed so that the outside of the foot is seen when seen from the viewpoint of the goalkeeper in most cases. Since the supporting foot faces the destination of the ball, watching the direction of the player's supporting foot is considered useful from the viewpoint of the goalkeeper. In addition, since supporting foot angles are different among kickers the goalkeeper may carefully watch the kicking habits of the kickers of the opposite team at normal times to apply them in games.

(3) When the pelvis is tilted rightward, the probability for the ball to go leftward is high and the opposite is the same as well. This can be regarded to be a natural kinesiological function to maintain the balance of the human body and rebound the trunk toward the trunk toward the destination of the ball in order to deliver more momentum.

(4) Shoulder angles are helpful for the prediction of ball directions in advance because ball directions can be predicted at the moment when the kicking foot reached the highest height already before the moment of kicking through shoulder tilt angles. Whereas the pelvic tilt angle is determined at the final moment of shooting since the ball is kicked using the turning force obtained while the pelvis is turned, the shoulder angle can be said to be already determined during the run-up.

(5) It can be seen that kickers generally make penalty kicks with the trunk a little tilted. The anteversion/retroversion angles of the trunk can be said to be closely related to the 
differences in the anthropotomical structures of kicking players, the positions and angle distances of the supporting foot and the ball, and ball directions. In addition, the angles may vary with the position of contact between the ball and the foot determined by kicking methods. The trunk tilt angle is determined by the angle of the kicking foot at the moment of hitting the ball, which can be said to affect the angle formed by the kicking foot and the ball. Therefore, goalkeepers and instructors that teach goal keepers can carefully watch kickers' penalty kick motions to prepare countermeasures and undergo training against the motions.

\section{Acknowledgments}

This study was supported by the Research Program funded by the Seoul National University of Science and Technology (2016).

\section{References}

[1] L. G. Cheong, H. S. Jin and M. C. Hyeon, "Comparison of penalty kick motions of elementary school soccer players according to the directions of the ball" The Korean Journal of the Elementary Physical Education, vol. 19, no. 4, (2014), pp. 197-205.

[2] L. G. Cheong, H. M. Ja and M. C. Hyeon, "The relationship between the kicker's motion before shooting and the ball direction in soccer penalty kicks" The Korean Journal of the Elementary Physical Education, vol. 13, no. 2, (2008), pp.141-154.

[3] L. J. Hun, "Science hidden in penalty kicks Sport" The Sport Kyunghyang Shinmun, (2014).

[4] L. B. Oh, "Kinematic analysis of soccer penalty kicks" Korean Journal of Sport Biomechanics, vol. 11, no. 2, (2001), pp.133-145.

[5] L. B. Oh, Y. J. Man, "Kinematic analysis of soccer inside penalty kicks" The Korean Journal of Physical Education, vol. 41, no. 4, (2002), pp. 541-547.

[6] S. S. Gwang, "Kinematic analysis of penalty kick motions for goalkeeper's prediction of ball directions" Master's thesis, Graduate School of Korea National Sport University, (2002).

[7] S. J. Mu, K. J. Jeong, P. H. Rim and K. S. Seon, "Kinematic analysis of lower extremity segments according to changes in goals during soccer inside penalty kick motions", Korean Journal of Sport Biomechanics, vol. 23, no. 2, (2013), pp.117-123.

[8] Y. I. A. Aziz and H. M. Karara, "Direct Linear Transformation from comparator Coordinates into Object Space Coordinates in Close-Range Photogrammetry" Proceedings of the Symposium on Close Range Photogrammetry, (1971).

[9] E. M. Bar and A. Friedman, "Psychological stress in soccer. the case of penalty kicks", Soccer-journal(New york), vol. 33, no. 6, (1988), pp. 49-52.

[10] W. S. Chae, N. J. Kang, J. W. Kim, C. J. Yun, S. D. Chae, C. H. Seok, G. Y. Park, and Y. T. Lim, "The Effect of upper Extremity Usage on Soccer Instep Kick Motion" Korean journal of sport Biomechanics, vol. 20, no. 1, (2010), pp. 41-47.

[11] P. H. Cho, "The Analysis of Transfer Angular Momentum on Lower Extremity during Place kicking in soccer" Korean Society of Leisure Sport, vol. 17, no. 1, (2006), pp. 423-430.

[12] FIFA Worild Cup: Comparative Statistics. www.fifa.com

[13] B. D. Hale and A. Whitehouse, "The effects of im-agery-manipulated appraisal on intensity and direction of competitive anxiety", Sport- psy-cholosgist-(Champaigu,III), vol. 12, no. 1, (1998), pp. 4051.

[14] Y. S. Kim, "Comparative analysis of Electromyography of the Lower extremities according to the changes of angles of approach during instep shooting of soccer" The Korean Journal of Physical Education, vol. 45, no. 3, (2006), pp. 623-632.

[15] T. McGarry and I. M. Franks, "On winning the penalty shoot-out in soccer", Journal-of-sports-sciences(London), vol. 18, no. 6, (2000), pp. 401-409.

[16] Plagenhoef, “Anatomical data for analyzing hu-mab motion”, Research Quarterly, (1971).

[17] J. M. Shin, "3-D Kinematics Comparative Analysis of Pen-alty Kick between Novice and Expert Soccer Players", Korean Journal of Sport Biomechanics, vol. 15, no. 4, (2005), pp. 13-24.

[18] Tant, "Segmental interactions of a three-dimen-sional soccer instep kick motion", Ph. D. Dissertation. Texas Weman's University, (1990).

[19] A. M. Williams, "Perceptual skill in soccer. Implication for talent identification and development" journal of sports Science, vol. 18, (2000), pp. 737-750.

[20] P. Allard, I. A. F. Stokes and J. Blanchi, "Three dimensional analysis of human movement", Human Kinetics, Champaign, (1995), pp. 166-168.

[21] H. Anton, Elementary Linear Algebra 8th. John Wiley \& sons, inc. New York, NY, (2000).

[22] S. Barbour, "Penalty-kicks and sports psychology", Soccer - journal - (Binghamton, N.Y.), vol. 37, no. 1, (1992), pp. 47-52 
[23] S. K. Harle and J. N. Vickers, "Training quiet eye improves accuracy in the basketball free throw. The Sport Psychologist, vol. 15, (2001), pp. 289-305.

[24] H. Inklaar, "Soccer injuries: Incidence and severity", Sports Med. 18, (1994), pp.55-73.

[25] Y. W. Jin, J. Y. Choi and J. M. Shin, "Kinematic Comparison of Three Soccer Shooting Motion", The Korean Journal of Physical Education, vol. 38, no. 4, (1999), pp. 537-547

[26] T. Kato and T. Fukuda, "Visual search strategies of baseball batters: Eye movements during the preparatory phase of batting", Perceptual and Motor Skills, vol. 94, (2002), pp. 380-386.

[27] W. Kuhn, "Penalty-kick strategies for shooters and goalkeepers", In, Reilly, et al. (eds.), Science and football: proceedings of the First World Congress of Science and Football, Liverpool, (1987), pp. 489492.

[28] Y. H. Kwon, KWON3D. Motion Analysis Package Version 3.1., (2005).

[29] L. S. Miller and S. E. Miller, "Anticipation of Professional soccer goalkeepers when facing right- and left-footed penalty kicks", Perceptual - and - motor - skills - (Missoula, Mont.), vol. 82, no. 3 Part 1, (1996), pp. 931-934.

[30] F. E. Rafael, S. F. Glenn, W. B. Steven, Z. Naiquan and R. A. James, "Kinematic comparisons of throwing different types of baseball pitches. Journal of applied biomechanics", (1998), pp. 1-23.

[31] J. M. Yoon, "Sport Biomechanical Analysis on Kicks in Soccer", Korean Journal of Sport Biomechanics, vol. 9, no. 20, (2000), pp. 117-128

\section{Author}

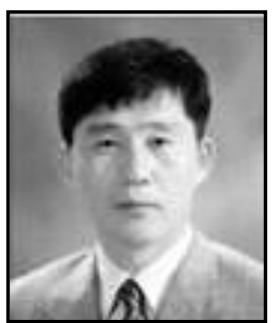

Chong-hoon Lee, he is in the Department of Sports Science, Seoul National University of Science \& Technology, 138 Gongreunggil, Gongreung2-dong 172, 139-743, leejh36@snut.ac.kr 